\title{
Adaptive Dynamic Programming based Control Scheme for Uncertain Two-Wheel Robots
}

\author{
Thien Van Nguyen and Hai Xuan Le \\ Hanoi University of Industry \\ Hanoi, Vietnam \\ nguyenthien@haui.edu.vn; hailx@haui.edu.vn \\ Minh Ngoc Nguyen \\ Hanoi University of Science and Technology \\ Hanoi, Vietnam \\ minhkenzin123@gmail.com
}

\author{
Hoang Viet Tran and Duc Anh Nguyen \\ Hanoi University of Science and Technology \\ Hanoi, Vietnam \\ \{hoangprohp1999,anhduc180198\}@gmail.com \\ Linh Nguyen \\ Federation University Australia \\ VIC, Australia \\ 1.nguyen@federation.edu.au
}

\begin{abstract}
The paper addresses the problem of effectively controlling a two-wheel robot given its inherent non-linearity and parameter uncertainties. In order to deal with the unknown and uncertain dynamics of the robot, it is proposed to employ the adaptive dynamic programming, a reinforcement learning based technique, to develop an optimal control law. It is interesting that the proposed algorithm does not require kinematic parameters while finding the optimal state controller is guaranteed. Moreover, convergence of the optimal control scheme is theoretically proved. The proposed approach was implemented in a synthetic two-wheel robot where the obtained results demonstrate its effectiveness.
\end{abstract}

Index Terms-Reinforcement learning, two-wheel robot, adaptive control, adaptive dynamic programming

\section{INTRODUCTION}

Two-wheel self-balancing vehicles have been increasingly become a popular alternative transportation method in recent years due to its flexibility during travelling in metropolitan areas [1]. Technically, the vehicle is built based on a two-wheel robot platform, which is well known in the control community due to its unstable dynamic performance and strong nonlinearity. That is, effectively and robustly controlling a twowheel robot given its uncertainty is a fundamental but still challenging problem.

In the control theory, sliding model control is widely used in systems highly requiring robustness [2], [3]. Nevertheless, the sliding model control assumes that a dynamic model of a system is deterministic and known, which is impractical [4], [5]. Recent researches show trend of adaptively learning system dynamics during controlling the system [6], [7]. One of good strategies for learning dynamic behaviours of intelligent robots is using reinforcement learning (RL) [8], which practically simulates learning ability of animals while interacting with environment to adapt and survive. RL has been used in variety of real-time optimal control methods such as adaptive dynamic programming (ADP) [8], where system dynamics are not exactly known. For instance, the authors in [9] exploited the policy iteration (PI) algorithm to eliminate internal kinematic models in optimally control an uncertain nonlinear system. In [10], Vrabie et al. presented a control design using RL to solve the linear quadratic regulator problem online. Another PI based method was developed in [11] with assumption of a full model-free system, where noise signals are proposed to add to input signals during learning process.

In this paper, we propose to exploit the ADP to model uncertain and nonlinear systems such as a two-wheel robot, which allows a control scheme to eliminate kinematic components in the system dynamic models. In other words, the dynamic parameters can be adaptively learned over time. We then employ the PI to design an efficient optimal control law for those systems, where its convergence is theoretically proved. The proposed approach was implemented and verified in a two-wheel robot in a synthetic environment in which the obtained results are highly promising.

The remaining of the paper is arranged as follows. Section II introduces the adaptive dynamic programming model before the proposed algorithm is discussed in Section III. Implementation of the control law in a two-wheel robot is presented in Section IV, where the obtained results are demonstrated. Conclusions are drawn in Section V.

\section{Adaptive Dynamic Programming Model}

Let us consider a continuous linear system as follows,

$$
\dot{x}=A x+B u,
$$

where $x \in R^{n}$ is a state vector of the system, $u \in R^{m}$ is a signal control vector, $A \in R^{n \times n}$ and $B \in R^{m \times m}$ are uncertainty matrices. Assume that the system is stable, there exist a matrix $\mathrm{K}$ so that A-BK is a Hurwitz matrix. That is, a full linear state feedback controller must be in the form

$$
u=-K x,
$$

which holds the following cost function

$$
J\left(x_{0}, u\right)=\int_{0}^{\infty}\left(x^{T} Q x+u^{T} R u\right) d t=x^{T} P x,
$$


where $Q=Q^{T} \geq 0$ and $R=R^{T} \geq 0$. Hence, $\left(A, Q^{1 / 2}\right)$ is observable. If both matrices $\mathrm{A}$ and $\mathrm{B}$ are completely identified, solution to a linear quadratic regulator (LQR) problem is equivalent to that of Riccati equation [12]

$$
A^{T} P+P A+Q-P B R^{-1} B^{T} P=0 .
$$

The equation has a uniquely positive symmetric solution $P^{*}$. Thus, the coefficient matrix of the optimal state feedback controller $K^{*}$ is computed as follows,

$$
K^{*}=R^{-1} B^{T} P^{*} \text {. }
$$

Suppose that $u_{K}(x)=-K x$ is an arbitrary feedback law. If $u_{K}$ is applied to the system (1), the cost function (3) can be written

$$
J\left(x_{0} ; u_{K}\right)=x_{0}^{T} P_{k} x_{0},
$$

where $P_{k}$ is defined as a cost matrix associated with the feedback gains $\mathrm{K}$ and given by

$$
P_{k}=\int_{0}^{\infty} e^{(A-B K)^{T} t}\left(Q+K^{T} R K\right) e^{(A-B K) t} d t .
$$

$P_{k}$ is finite iff it is a Hurwitz matrix. That is, $P_{k}$ is the unique (positive definite) solution of the linear equation

$$
(A-B K)^{T} P+P(A-B K)+Q+K^{T} R K=0 .
$$

Moreover, if $K_{1}$ and $K_{2}$ are gain matrices associated with the cost matrices $P_{1}$ and $P_{2}$, it can be shown that

$$
\begin{aligned}
P_{1}-P_{2} & =\int_{0}^{\infty} e^{\left(A-B K_{2}\right)^{T} t}\left[\left(K_{1}-K_{2}\right)^{T} R\left(K_{1}-K_{2}\right)\right. \\
& -\left(K_{1}-K_{2}\right)^{T}\left(B^{T} P_{1}-R K_{2}\right) \\
& \left.-\left(B^{T} P_{1}-R K_{2}\right)^{T}\left(K_{1}-K_{2}\right)\right] e^{\left(A-B K_{2}\right) t} d t .
\end{aligned}
$$

or

$$
\begin{aligned}
P_{1}-P_{2} & =\int_{0}^{\infty} e^{\left(A-B K_{1}\right)^{T} t}\left[\left(K_{1}-K_{2}\right)^{T} R\left(K_{1}-K_{2}\right)\right. \\
& -\left(K_{1}-K_{2}\right)^{T}\left(B^{T} P_{2}-R K_{2}\right) \\
& \left.-\left(B^{T} P_{2}-R K_{2}\right)^{T}\left(K_{1}-K_{2}\right)\right] e^{\left(A-B K_{1}\right) t} d t .
\end{aligned}
$$

Theorem 1. If $K_{0} \in R^{m \times n}$ is a matrix of the state feedback controller (1). The positive symmetric solution $P_{k}$ of the Lyapunov equation

$$
\left(A-B K_{k}\right)^{T} P_{k}+P_{k}\left(A-B K_{k}\right)+Q+K_{k} R K_{k}^{T}=0,
$$

can be computed as follows,

$$
\begin{aligned}
K_{k} & =R^{-1} B^{T} P_{k-1} \\
A_{k} & =A-B K_{k},
\end{aligned}
$$

where $K_{1}$ is chosen such that $A_{1}=A-B K_{1}$ is a Hurwitz matrix. And,

1. $A_{k}=A-B K_{k}$ is a Hurwitz matrix.

2. $P^{*} \leq P_{K+1} \leq P_{k}$.
3. $\lim _{n \rightarrow \infty} K_{k}=K^{*}, \lim _{n \rightarrow \infty} P_{k}=P^{*}$.

$\stackrel{n \rightarrow \infty}{\text { Proof: }}$. Let $P_{1}$ be a cost matrix associated with $K_{1} . P_{1}$ then satisfies (11) with $\mathrm{k}=1$. Now set $K_{2}=R^{-1} B^{T} P_{1}$ and let $P_{2}$ be its associated cost matrix. Using (10) we obtain

$$
P_{1}-P_{2}=\int_{0}^{\infty} e^{A_{2} T}\left(K_{1}-K_{2}\right)^{T} R\left(K_{1}-K_{2}\right) e^{A_{2} t} d t \geq 0
$$

so that $P_{2} \leq P_{1}$. In addition, By using (10),

$$
P_{2}-P^{*}=\int_{0}^{\infty} e^{A_{2}{ }^{T} t}\left(K_{2}-K^{*}\right)^{T} R\left(K_{2}-K^{*}\right) e^{A_{2} t} d t \geq 0 .
$$

Hence $P_{2}$ is lower bounded has a finite norm. In other words, $A_{2}$ is a Hurwitz matrix. The result can be extended for $k=$ $3,4, \ldots$.

2. Obviously, $P^{*} \leq P_{2} \leq P_{1}$ holds when $\mathrm{k}=1$. The result can be extended for $k=2,3, \ldots$.

3. $\lim _{k \rightarrow \infty} P_{k}=P_{\infty}$ holds, presented by the theorem on the monotonic convergence of positive operators [13]. By calculating limit of (11) as $k \rightarrow \infty$ one obtains

$$
A^{T} P_{\infty}+P_{\infty} A+Q-P_{\infty} B R^{-1} B^{T} P_{\infty}=0 .
$$

Since $P^{*}$ is the unique positive definite solution of (13), $P_{\infty}=$ $P^{*}$. Hence $\lim _{k \rightarrow \infty} P_{k}=P^{*}$. Given (12), it yields $\lim _{k \rightarrow \infty} K_{k}=$ $K^{*}$.

\section{PROPOSED APPROACH}

In order to solve (4) without using the matrix A, an approximate solution can be obtained through the policy iteration (PI) technique [10]. In that case, (3) can be rewritten as follows,

$$
\begin{aligned}
& x^{T}(t) P_{k} x(t)-x^{T}(t+T) P_{k} x(t+T) \\
& =\int_{t}^{t+T}\left(x^{T} Q x+u_{k}^{T} R u_{k}\right) d t,
\end{aligned}
$$

where $u_{k}=-K_{k} x$ is the state feedback control law in time domain $[t, t+T]$. From (14), $P_{k}$ can be approximated without requiring A. However, persistent excitation needs to be guaranteed so that $P_{k}$ is unique. To address the issue, adding probe noise to the state feedback control signal was proposed in [11]. Thus, the system model (1) is rewritten by

$$
\dot{x}=A_{k} x+B\left(K_{k} x+u\right),
$$

where $A_{k}=A-B K_{k}$, which can be used to represent (14) as follows,

$$
\begin{aligned}
& x^{T}(t+T) P_{k} x(t+T)-x^{T}(t) P_{k} x(t) \\
& =\int_{t}^{t+T}\left[x^{T}\left(A_{k}^{T} P_{k}+P_{k} A_{k}\right) x+2\left(u+K_{k} x\right)^{T} B^{T} P_{k} x\right] d t \\
& =-\int_{t}^{t+T} x^{T} Q_{k} x d t+2 \int_{t}^{t+T}\left(u+K_{k} x\right)^{T} R K_{k+1} x d t,
\end{aligned}
$$

where $Q_{k}=Q+K_{k}^{T} R K_{k}$. And the control signal is added by a small noise,

$$
u=-K_{k} x+e
$$


Let us define $P \in R^{n \times n} \rightarrow \hat{P} \in R^{n \times(n+1) / 2}$ and $x \in R^{n} \rightarrow$ $\bar{x} \in R^{n \times(n+1) / 2}$, where

$$
\begin{aligned}
& \hat{P}=\left[p_{11}, 2 p_{12}, \ldots, 2 p_{1 n}, p_{22}, 2 p_{23}, \ldots, 2 p_{n-1, n}, p_{n n}\right]^{T} \\
& \bar{x}=\left[x_{1}^{2}, x_{1} x_{2}, \ldots, x_{1} x_{n}, x_{2}^{2}, x_{2} x_{3}, \ldots, x_{n-1} x_{n}, x_{n}^{2}\right]^{T} .
\end{aligned}
$$

By using Kronecker product one has

$$
\begin{aligned}
x^{T} Q_{k} x= & \left(x^{T} \otimes x^{T}\right) \operatorname{vec}\left(Q_{k}\right) \\
\left(u+K_{k} x\right) R K_{k+1} x= & {\left[\left(x^{T} \otimes x^{T}\right)\left(I_{n} \otimes K_{k}^{T} R\right)\right.} \\
& \left.+\left(x^{T} \otimes u^{T}\right)\left(I_{n} \otimes R\right)\right] \operatorname{vec}\left(K_{k+1}\right)
\end{aligned}
$$

If considering (16) in some time domains $\left[t_{0}, t_{1}\right],\left[t_{1}, t_{2}\right], \ldots\left[t_{l-1}, t_{l}\right]$, one has $l$ equations. We define matrices $\delta_{x x} \in R^{l \times \frac{n(n+1)}{2}}, I_{x x} \in R^{l \times n^{2}}, I_{x u} \in R^{l \times m n}$ as follows

$$
\begin{aligned}
\delta_{x x} & =\left[\bar{x}\left(t_{0}\right)-\bar{x}\left(t_{1}\right), \bar{x}\left(t_{1}\right)-\bar{x}\left(t_{2}\right), \ldots, \bar{x}\left(t_{l-1}\right)-\bar{x}\left(t_{l}\right)\right]^{T} \\
I_{x x} & =\left[\int_{t_{0}}^{t_{1}} x \otimes x d t, \int_{t_{1}}^{t_{2}} x \otimes x d t, \ldots, \int_{t_{l-1}}^{t_{l}} x \otimes x d t\right]^{T} \\
I_{x u} & =\left[\int_{t_{0}}^{t_{1}} x \otimes u d t, \int_{t_{1}}^{t_{2}} x \otimes u d t, \ldots, \int_{t_{l-1}}^{t_{l}} x \otimes u d t\right]^{T} .
\end{aligned}
$$

$l$ equations can be presented in a matrix format.

$$
\Theta_{k}\left[\begin{array}{c}
\hat{P}_{k} \\
\operatorname{vec}\left(K_{k+1}\right)
\end{array}\right]=\Xi_{k},
$$

where $\Theta_{k} \in R^{\mathrm{l} \times n(n+1)+m n}$ and $\Xi_{k} \in R^{l}$ are specified by

$$
\begin{aligned}
& \Theta_{k}=\left[\delta_{x x}, 2 I_{x x}\left(I_{n} \otimes K_{k}^{T} R\right)+2 I_{x u}\left(I_{n} \otimes R\right)\right] \\
& \Xi_{k}=I_{x x} \operatorname{vec}\left(Q_{k}\right)
\end{aligned}
$$

When $\Theta_{k}$ is a full rank column matrix, equation (18) can be solved by using the least squares method

$$
\left[\begin{array}{c}
\hat{P}_{k} \\
\operatorname{vec}\left(K_{k+1}\right)
\end{array}\right]=\left(\Theta_{k}^{T} \Theta_{k}\right)^{-1} \Theta_{k}^{T} \Xi_{k}
$$

It is noted that the probe noise signal in this work is selected as sum of sine signals,

$$
e=0.1 \sum \sin \left(\omega_{i} t\right)
$$

where $\omega_{i}$ is frequency randomly selected in $[-500,500] \mathrm{Hz}$. Convergence of the proposed algorithm is provided in the following theorem, which is derived from Kleinman's theorem 1 [14].

Theorem 2. Given the initial stable controller $K_{0} \in R^{m \times n}$ and $\Theta_{k}$ is full rank, the sequence matrices $\left\{P_{i}\right\}_{i=0}^{\infty}$ and $\left\{K_{i}\right\}_{i=0}^{\infty}$ obtained from the equation (19) converge to the optimal values $P^{*}$ and $K^{*}$.

Proof: Given the state feedback controller $K_{k}$, if $P_{k}^{T}=$ $P_{k}$ is the solution of (11) and $K_{k+1}$ is identified by (12), then from (16) $P_{k}$ and $K_{k+1}$ satisfy (19). On the other hand, if $\Theta_{k}$ is full rank, the solution of (19) is unique. Hence, (19) is equivalent to (11) and (12). In other words, the optimal algorithm based on the adaptive dynamic programming is equivalent to the iterative method in the theorem 1 .
TABLE I: PARAMETERS OF TWO-WHEEL ROBOTS.

\begin{tabular}{|l|l|}
\hline Symbol & Definition \\
\hline $\mathrm{x}$ & Position of two-wheel self-balancing vehicle \\
\hline$\theta$ & Inclined angle of vehicle body \\
\hline$\psi$ & Directional angle of the vehicle \\
\hline $\mathrm{d}$ & $\begin{array}{l}\text { Distance between the center of vehicle body to the } \\
\text { axle connecting the two wheels of the car }\end{array}$ \\
\hline $\mathrm{l}$ & Radius of the vehicle's wheel \\
\hline $\mathrm{r}$ & Mass of the vehicle body \\
\hline$m_{B}$ & Mass of left wheel (right wheel) \\
\hline$m_{W}$ & Mass of the vehicle body \\
\hline $\mathrm{J}$ & $\begin{array}{l}\text { Moment of inertia of vehicle wheel corresponding to } \\
\text { the axle of wheel }\end{array}$ \\
\hline $\mathrm{K}$ & $\begin{array}{l}\text { Moment of inertia of vehicle wheel corresponding to } \\
\text { vertical axle }\end{array}$ \\
\hline$K_{m}$ & Torque \\
\hline$i_{L}, i_{R}$ & $\begin{array}{l}\text { Current flows through the motor of the left wheel } \\
\text { (right wheel) }\end{array}$ \\
\hline$T_{L}, T_{R}$ & Torque of motor of left wheel (right wheel) \\
\hline$\gamma_{L}, \gamma_{R}$ & Rotational angle of left wheel (right wheel) \\
\hline$c_{\alpha}$ & Coefficient of friction on wheel's axle \\
\hline$I_{1}, I_{2}, I_{3}$ & $\begin{array}{l}\text { Moment of inertia of vehicle body corresponding to } \\
\text { the reference system } \mathrm{B}\end{array}$ \\
\hline$(L, R, B)$ & Left wheel, Right wheel, Vehicle Body \\
\hline$\hat{\mathrm{w}}^{L}, \hat{\mathrm{w}}^{R}, \hat{\mathrm{w}}^{B}$ & Angular velocity of (L, R, B) \\
\hline$\hat{v}^{L}, \hat{v}^{R}, \hat{v}^{B}$ & Velocity of center (L, R, B) \\
\hline$\hat{v}^{C}, \hat{\mathrm{w}}^{C}$ & $\begin{array}{l}\text { Translational velocity and angular velocity in } \\
\text { reference system C }\end{array}$ \\
\hline
\end{tabular}

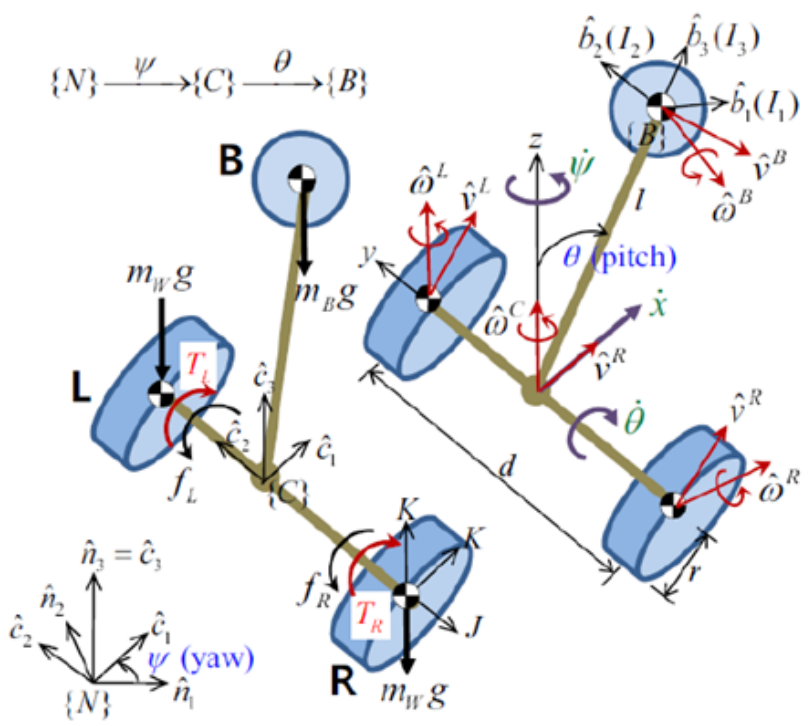

Fig. 1: Model of a two-wheel robot.

\section{EVAluation ON Two-WheEl Robots}

\section{A. Two-Wheel Robot Model}

The model of a two-wheel robot is illustrated in Fig. 2, where its parameters are explained in Table 1. It is noted that the coefficient transfer matrices between some reference 
systems are given by

$$
\begin{array}{r}
{ }^{N} R_{C}(\psi)=\left[\begin{array}{ccc}
\cos \psi & -\sin \psi & 0 \\
\sin \psi & \cos \psi & 0 \\
0 & 0 & 1
\end{array}\right], \\
{ }^{C} R_{B}(\theta)=\left[\begin{array}{ccc}
\cos \theta & 0 & \sin \theta \\
0 & 1 & 0 \\
-\sin \theta & 0 & \cos \theta
\end{array}\right],
\end{array}
$$

The coordinates of its body, left and right wheels in the reference system $\mathrm{N}$ are identified as follows,

$$
\begin{aligned}
& x_{B}=x_{C}+l \sin \theta \cos \psi, y_{B}=y_{C}+l \sin \theta \sin \psi, \\
& z_{B}=l \cos \theta, x_{L}=x_{C}-\frac{l}{2} \sin \psi, y_{L}=y_{C}+\frac{d}{2} \cos \psi, \\
& x_{R}=x_{C}+\frac{d}{2} \sin \psi, y_{R}=x_{C}+\frac{d}{2} \cos \psi
\end{aligned}
$$

The nonholonomic equations in the equivalent system can be presented by

$$
\left\{\begin{array}{l}
\dot{x}_{L} \cos \psi+\dot{y}_{L} \sin \psi=r \dot{\gamma}_{L} \\
\dot{x}_{R} \cos \psi+\dot{y}_{R} \sin \psi=r \dot{\gamma}_{R} \\
\left(\dot{x}_{L}+\dot{x}_{R}\right) \sin \psi=\left(\dot{y}_{L}+\dot{y}_{R}\right) \cos \psi
\end{array},\right.
$$

which is equivalent to

$$
\left\{\begin{array}{l}
\dot{x}_{C} \cos \psi+\dot{y}_{C} \sin \psi-\frac{d}{2} \dot{\psi}-r \dot{\gamma}_{L}=0 \\
\dot{x}_{C} \cos \psi+\dot{y}_{C} \sin \psi+\frac{d}{2} \dot{\psi}-r \dot{\gamma}_{R}=0 \\
\dot{x}_{C} \sin \psi-\dot{y}_{C} \cos \psi=0
\end{array}\right.
$$

Hence, the translational velocity of the robot is

$$
\begin{gathered}
\dot{x}=\dot{x}_{C} \cos \psi+\dot{y}_{C} \sin \psi \\
\dot{\gamma}_{L}=\frac{\dot{x}-d / 2 \dot{\psi}}{r}, \dot{\gamma}_{R}=\frac{\dot{x}+d / 2 \dot{\psi}}{r} .
\end{gathered}
$$

Taking derivatives of (23) leads to the translational velocities of the left and right wheels and the vehicle body as follows,

$$
\begin{aligned}
& \hat{v}^{L}=\left(\dot{x}_{C}-\frac{d}{2} \dot{\psi} \cos \psi\right) \hat{n}_{1}+\left(\dot{y}_{C}-\frac{d}{2} \dot{\psi} \sin \psi\right) \hat{n}_{2} \\
& \hat{v}^{R}=\left(\dot{x}_{C}+\frac{d}{2} \dot{\psi} \cos \psi\right) \hat{n}_{1}+\left(\dot{y}_{C}+\frac{d}{2} \dot{\psi} \sin \psi\right) \hat{n}_{2} \\
& \hat{v}^{B}=\left(\dot{x}_{C}+l \dot{\theta} \cos \theta \cos \psi-l \dot{\psi} \sin \theta \sin \psi\right) \hat{n}_{1} \\
& +\left(\dot{y}_{C}+l \dot{\theta} \cos \theta \sin \psi+l \dot{\psi} \sin \theta \cos \psi\right) \hat{n}_{2}-(l \dot{\theta} \sin \theta) \hat{n}_{3} .
\end{aligned}
$$

From Fig. 2, angular velocities of the left and right wheels and the vehicle body can be computed by

$$
\begin{aligned}
& \hat{\mathrm{w}}^{L}=\hat{\mathrm{w}}^{C}+\dot{\gamma}_{L} \hat{c}_{2}=\dot{\psi} \hat{c}_{3}+\frac{1}{r}\left(\dot{x}-\frac{d \dot{\psi}}{2}\right) \hat{c}_{2}, \\
& \hat{\mathrm{w}}^{R}=\hat{\mathrm{w}}^{C}+\dot{\gamma}_{R} \hat{c}_{2}=\dot{\psi} \hat{c}_{3}+\frac{1}{r}\left(\dot{x}+\frac{d \dot{\psi}}{2}\right) \hat{c}_{2}, \\
& \hat{\mathrm{w}}^{B}=\hat{\mathrm{w}}^{C}+\dot{\theta} \hat{b}_{2}=(-\dot{\psi} \sin \theta) \hat{b}_{1}+\dot{\theta} \hat{b}_{2}+(\dot{\psi} \cos \theta) \hat{b}_{3} .
\end{aligned}
$$

On the other hand, the translational velocities of the left and right wheels and the vehicle body can be computed by the other methods,

$$
\begin{aligned}
& \hat{v}^{L}=\dot{x} \hat{c}_{1}+\hat{w}^{C} \frac{d}{2} \hat{c}_{2}=\left(\dot{x}-\frac{d \dot{\psi}}{2}\right) \hat{c}_{1}, \\
& \hat{v}^{R}=\dot{x} \hat{c}_{1}+\hat{w}^{C}-d \\
& \hat{v}_{2}=\left(\dot{x}+\frac{d \dot{\psi}}{2}\right) \hat{c}_{1}, \\
& \hat{v}^{B}=\dot{x} \hat{c}_{1}+\hat{w}^{B} l \hat{b}_{3}=\dot{x} \hat{c}_{1}+(l \dot{\theta}) \hat{b}_{1}+(l \dot{\psi} \sin \theta) \hat{b}_{2},
\end{aligned}
$$

which leads to the translational kinetic energy of the robot

$$
T_{\text {trans }}=\frac{1}{2} m_{W}\left(\hat{v}^{L} \hat{v}^{L}\right)+\frac{1}{2} m_{W}\left(\hat{v}^{R} \hat{v}^{R}\right)+\frac{1}{2} m_{B}\left(\hat{v}^{B} \hat{v}^{B}\right) .
$$

Moreover, the rotational kinetic energy is calculated by

$$
T_{\text {rot }}=\frac{1}{2}\left(\hat{w}^{L}\right)^{T} \hat{I}_{L} \hat{w}^{L}+\frac{1}{2}\left(\hat{w}^{R}\right)^{T} \hat{I}_{R} \hat{w}^{R}+\frac{1}{2}\left(\hat{w}^{B}\right)^{T} \hat{I}_{B} \hat{w}^{B} .
$$

Replacing (25) to (28) yields

$T_{\text {rot }}=\frac{1}{2}\left(\left(I_{1}-I_{3}\right) \sin ^{2} \theta+I_{3}+2 K\right) \dot{\psi}^{2}+\frac{1}{2} I_{2} \dot{\theta}^{2}+\frac{1}{2} J\left(\dot{\gamma}_{L}^{2}+\dot{\gamma}_{L}^{2}\right)$

It is noticed that the potential energy of the robot is given by

$$
V=m_{B} g l \cos \theta .
$$

Thus, the Lagrange function is formulated as follows,

$$
L=T-V=T_{\text {trans }}+T_{\text {rot }}-V
$$

If we define $q_{1}=x_{C}, q_{2}=y_{C}, q_{3}=\theta, q_{4}=\psi, q_{5}=\gamma_{L}, q_{6}=$ $\gamma_{R}$, the kinetic function Lagrange II for the two-wheel robots can be given by

$\frac{d}{d t}\left(\frac{\partial L}{\partial \dot{q}_{i}}\right)-\frac{\partial L}{\partial \dot{q}_{i}}=Q_{i}+\sum_{j} \lambda_{j} a_{i j}(i=1,2, \ldots, 6, j=1,2,3)$,

where

$$
\begin{aligned}
& a_{1 i}=\frac{\partial}{\partial \dot{q}_{i}}\left(\dot{x}_{C} \cos \psi+\dot{y}_{C} \sin \psi-\frac{d}{2} \dot{\psi}-r \dot{\gamma}_{L}\right) \\
& \left.a_{2 i}=\frac{\partial}{\partial \dot{q}_{i}} \dot{x}_{C} \cos \psi+\dot{y}_{C} \sin \psi+\frac{d}{2} \dot{\psi}-r \dot{\gamma}_{R}\right) \\
& a_{1 i}=\frac{\partial}{\partial \dot{q}_{i}}\left(\dot{x}_{C} \sin \psi-\dot{y}_{C} \cos \psi\right)
\end{aligned}
$$

Here are steps to solve (34).

Step 1: $x_{C}$

$$
\begin{aligned}
& \mathrm{m}_{B}\left(\begin{array}{l}
\ddot{x}_{C}+l \ddot{\theta} \cos \theta \cos \psi-l \ddot{\psi} \sin \theta \sin \psi \\
-2 l \dot{\theta} \dot{\psi} \cos \theta \sin \psi-l\left(\dot{\theta}^{2}+\dot{\psi}^{2}\right) \sin \theta \cos \psi
\end{array}\right) \\
& +2 m_{\mathrm{w}} \ddot{x}_{C}=\lambda_{1} \cos \psi+\lambda_{2} \cos \psi+2 \lambda_{3} \sin \psi .
\end{aligned}
$$

Step 2: $y_{C}$

$$
\begin{aligned}
& \mathrm{m}_{B}\left(\begin{array}{c}
\ddot{y}_{C}+l \ddot{\theta} \cos \theta \sin \psi+l \ddot{\psi} \sin \theta \cos \psi \\
+2 l \dot{\theta} \dot{\psi} \cos \theta \cos \psi-l\left(\dot{\theta}^{2}+\dot{\psi}^{2}\right) \sin \theta \sin \psi
\end{array}\right) \\
& +2 m_{\mathrm{w}} \ddot{y}_{C}=\lambda_{1} \sin \psi+\lambda_{2} \sin \psi-2 \lambda_{3} \cos \psi .
\end{aligned}
$$

Step 3: $\theta$

$$
\begin{aligned}
& \mathrm{m}_{B}\left(x_{C} \cos \psi+y_{C} \sin \psi\right) l \cos \theta+\left(I_{2}+m_{B} l^{2}\right) \theta \\
& -\left(I_{1}-I_{3}+m_{B} l^{2}\right) \dot{\psi}^{2} \sin \theta \cos \theta-m_{B} g l \sin \theta \\
& =-\left(T_{L}+T_{R}\right)+c_{\alpha}\left(\dot{\gamma}_{L}-\dot{\theta}\right)+c_{\alpha}\left(\dot{\gamma}_{R}-\dot{\theta}\right) .
\end{aligned}
$$

Step 4: $\psi$

$\left\{I_{3}+m_{W} d^{2} / 2+2 K+\left(I_{1}-I_{3}\right) \sin ^{2} \theta\right\} \ddot{\psi}$

$+m_{B}\left\{-\ddot{x}_{C} \sin \psi+\ddot{y}_{C} \cos \psi-\left(\dot{x}_{C} \cos \psi+\dot{y}_{C} \sin \psi\right) \dot{\psi}\right\}$

$l \sin \theta+m_{B}\left(\dot{x}_{C} \cos \psi+\dot{y}_{C} \sin \psi\right) \dot{\psi} l \sin \theta$

$+2\left(I_{1}-I_{3}\right) \dot{\theta} \dot{\psi} \sin \theta \cos \theta$

$=-\frac{d \lambda_{1}}{2}+\frac{d \lambda_{2}}{2}$. 
Step 5: $\gamma_{L}$

$$
J \ddot{\gamma}_{L}=-r \lambda_{1}+T_{L}-c_{\alpha}\left(\dot{\gamma}_{L}-\dot{\theta}\right) .
$$

Step 6: $\gamma_{R}$

$$
J \ddot{\gamma}_{R}=-r \lambda_{1}+T_{R}-c_{\alpha}\left(\dot{\gamma}_{R}-\dot{\theta}\right) .
$$

Then we have

$$
T_{L}=K_{m} i_{L}, T_{R}=K_{m} i_{R}
$$

From (22), (23), (34) and (35), one has

$$
\begin{aligned}
& \quad\left\{m_{B}+2 m_{\mathrm{W}}+\frac{2 J}{r^{2}}\right\} \ddot{x}-m_{B} l\left(\dot{\theta}^{2}+\dot{\psi}^{2}\right) \sin \theta \\
& +\left(m_{B} l \cos \theta\right) \ddot{\theta}+\frac{2}{r} c_{\alpha}\left(\frac{\dot{x}}{r}-\dot{\theta}\right)=\frac{K_{m}\left(i_{L}+i_{R}\right)}{r} \\
& \quad\left(I_{2}+m_{B} l^{2}\right) \ddot{\theta}+\left(I_{3}-I_{1}-m_{B} l^{2}\right) \dot{\psi}^{2} \sin \theta \cos \theta \\
& \quad+\left(m_{B} l \cos \theta\right) \ddot{x}-m_{B} l \sin \theta-2 c_{\alpha}\left(\frac{\dot{x}}{r}-\dot{\theta}\right) \\
& \quad=-K_{m}\left(i_{L}+i_{R}\right), \\
& \left\{I_{3}+2 K+\left(m_{\mathrm{W}}+\frac{J}{r^{2}}\right) \frac{d^{2}}{2}-\left(I_{3}-I_{1}-m_{B} l^{2}\right) \dot{\psi}^{2} \sin ^{2} \theta\right\} \ddot{\psi} \\
& +\left\{m_{B} l \dot{x}-2\left(I_{3}-I_{1}-m_{B} l^{2}\right) \dot{\theta} \cos \theta\right\} \dot{\psi} \sin \theta+c_{\alpha} \dot{\psi} \frac{d^{2}}{2 r^{2}} \\
& =\left(i_{R}-i_{L}\right) K_{m} \frac{d}{2 r} .
\end{aligned}
$$

(40)

Now, we can obtain the kinetic equation for two-wheel robots as follows,

$$
\dot{x}=f(x)+g(x) u=F(x, u),
$$

where

$$
\begin{aligned}
& \dot{x}_{1}=x_{4}, \dot{\mathrm{x}}_{2}=x_{5}, \dot{\mathrm{x}}_{3}=x_{6}, \dot{\mathrm{x}}_{4}=F_{4}(x, u)=\frac{\Omega_{1}+\Omega_{2}}{\Omega} \\
& \dot{x}_{5}=F_{5}(x, u)=\frac{\Omega_{3}+\Omega_{4}}{\Omega}, \dot{x}_{6}=F_{6}(x, u)=\frac{\Omega_{5}}{\Omega_{6}} \\
& \Omega_{1}=r^{2}\left(m_{B} l^{2}+I_{2}\right)\left\{\begin{array}{l}
K_{m} \frac{u_{1}+u_{2}}{r}+\frac{2 c_{\alpha}}{r}\left(x_{5}-\frac{x_{4}}{r}\right) \\
+m_{B} l \sin \left(x_{2}\right)\left(x_{5}{ }^{2}+x_{6}{ }^{2}\right)
\end{array}\right\}, \\
& \Omega_{2}=m_{B} l r^{2} \cos \left(x_{2}\right)\left\{\begin{array}{l}
-\cos \left(x_{2}\right) \sin \left(x_{2}\right) \times \\
\left(m_{B} l^{2}+I_{1}-I_{3}\right) x_{6}^{2} \\
+K_{m}\left(u_{1}+u_{2}\right) \\
+2 c_{\alpha}\left(x_{5}-\frac{x_{4}}{r}\right) \\
-m_{B} g l \sin \left(x_{2}\right)
\end{array}\right\}, \\
& \Omega_{3}=\left[2 J+\left(\begin{array}{l}
\cos \left(x_{2}\right) \sin \left(x_{2}\right) \times \\
\left(m_{B} l^{2}+I_{1}-I_{3}\right) x_{6}^{2} \\
-K_{m}\left(u_{1}+u_{2}\right) \\
-2 c_{\alpha}\left(x_{5}-\frac{x_{4}}{r}\right) \\
+m_{B} g l \sin \left(x_{2}\right)
\end{array}\right\},\right. \\
& \Omega_{4}=-m_{B} l r^{2} \cos \left(x_{2}\right)\left\{\begin{array}{l}
K_{m} \frac{u_{1}+u_{2}}{r} \\
+\frac{2 c_{\alpha}}{r}\left(x_{5}-\frac{x_{4}}{r}\right) \\
+m_{B} g l \sin \left(x_{2}\right)\left(x_{5}^{2}+x_{6}^{2}\right)
\end{array}\right\}, \\
& \Omega_{5}=2 r^{2}\left\{\begin{array}{l}
-K_{m} d \frac{u_{1}+u_{2}}{2 r}-x_{6} \sin \left(x_{2}\right) \times \\
{\left[m_{B} l x_{4}+2 x_{5} \cos \left(x_{2}\right)\left(m_{B} l^{2}+I_{1}-I_{3}\right)\right]} \\
-\frac{c_{\alpha} d^{2} x_{6}}{2 r^{2}} \quad
\end{array}\right\}
\end{aligned}
$$

$$
\begin{aligned}
\Omega_{6}= & \left(2 I_{3}+4 K+m_{\mathrm{W}} d^{2}\right) r^{2} \\
& +\left(2\left(m_{B} l^{2}+I_{1}-I_{3}\right) \sin ^{2}\left(x_{2}\right)\right) r^{2}+J d^{2}, \\
\Omega= & \left(m_{B} l r\right)^{2}\left[1-\cos ^{2}\left(x^{2}\right)\right]+2 I_{2} J+2 J m_{B} l^{2} \\
& +\left(I_{2} m_{B}+2 I_{2} m_{\mathrm{W}}+2 m_{B} m_{\mathrm{W}} l^{2}\right) r^{2} .
\end{aligned}
$$

\section{B. Linearization of Two-Wheel Robots}

Assume that $x_{e}$ is a balanced point of a two-wheel robot. That is, $x_{e}=\left[\begin{array}{llllll}* & 0 & * & 0 & 0 & 0\end{array}\right]^{T}$ is the solution of $F\left(x_{e}, 0\right)=0$, where $*$ is a random value of $x_{1}$ and $x_{3}$. If $*=0, x_{e}=0$. Hence, linearizing the two-wheel robot model around $x_{e}$ is given by

$$
\dot{x}=A x+B u,
$$

where

$$
\begin{aligned}
& A=\left.\frac{\partial F}{\partial x}\right|_{x=x_{e}}=\left[\begin{array}{cccccc}
0 & 0 & 0 & 1 & 0 & 0 \\
0 & 0 & 0 & 0 & 1 & 0 \\
0 & 0 & 0 & 0 & 0 & 1 \\
0 & a_{42} & 0 & a_{44} & a_{45} & 0 \\
0 & a_{52} & 0 & a_{54} & a_{55} & 0 \\
0 & 0 & 0 & 0 & 0 & a_{66}
\end{array}\right] \\
& B=\left.\frac{\partial F}{\partial u}\right|_{x=x_{e}}=\left[\begin{array}{cc}
0 & 0 \\
0 & 0 \\
0 & 0 \\
b_{41} & b_{42} \\
b_{51} & b_{52} \\
b_{61} & b_{62}
\end{array}\right] \\
& a_{42}=\frac{-\left(m_{B} l r\right)^{2} g}{\Delta}, a_{44}=\frac{-2 c_{\alpha}\left(m_{B} l^{2}+I_{2}+m_{B} l r\right)}{\Delta}, \\
& a_{45}=\frac{2 c_{\alpha} r\left(m_{B} l^{2}+I_{2}+m_{B} l r\right)}{\Delta}, \\
& a_{52}=\frac{m_{B} g l\left(2 J+m_{B} r^{2}+2 m_{W} r^{2}\right)}{\Delta}, \\
& a_{54}=\frac{2 c_{\alpha} m_{B} l+2 c_{\alpha}\left(2 J+m_{B} r^{2}+2 m_{W} r^{2}\right) / r}{\Delta}, \\
& a_{55}=\frac{-2 c_{\alpha} m_{B} l r-2 c_{\alpha}\left(2 J+m_{B} r^{2}+2 m_{W} r^{2}\right)}{\Delta}, \\
& a_{66}=\frac{-c_{\alpha} d^{2}}{J d^{2}+\left(2 I_{3}+4 K+2 d^{2}\right) r^{2}}, \\
& b_{41}=b_{42}=\frac{K_{m} r\left(m_{B} l^{2}+I_{2}+m_{B} l r\right)}{\Delta} \\
& b_{51}=b_{52}=\frac{-K_{m}\left(2 J+m_{B} r^{2}+2 m_{W} r^{2}+m_{B} l r\right)}{\Delta} \text {, } \\
& b_{61}=-b_{62}=\frac{-K_{m} d r}{J d^{2}+\left(2 I_{3}+4 K+m_{W} d^{2}\right) r^{2}}, \\
& \Delta=2 I_{2} J+2 J m_{B} l^{2}+I_{2} m_{B} r^{2}+2 I_{2} m r^{2}+2 M l^{2} m r^{2} .
\end{aligned}
$$

\section{Simulation Results}

To demonstrate effectiveness of the proposed approach, we implemented it in controlling a two-wheel robot in a synthetic environment, where the robot parameters were set as follows. $m_{B}=0.5(\mathrm{~kg}), m_{W}=0.04\left(\mathrm{~kg} \cdot \mathrm{m}^{2}\right), l=0.08(\mathrm{~m})$, $d=0.16(\mathrm{~m}), r=0.033(\mathrm{~m}), g=9.81\left(\mathrm{~m} / \mathrm{s}^{2}\right), c_{\alpha}=$ 


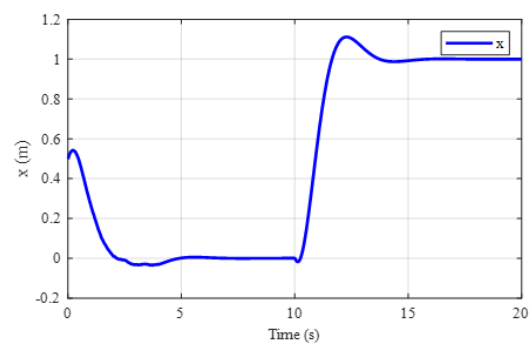

(a) Trajectory of the robot.

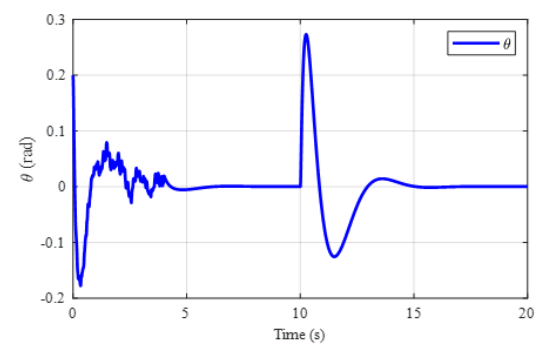

(b) Inclined angle $\theta$ of the robot.

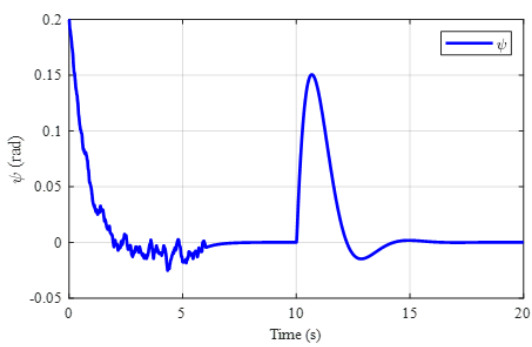

(c) Directional angle $\psi$ of the robot.

Fig. 2: Two-wheel robot simulation results obtained by the proposed algorithm.

$5.10-4(\mathrm{Ns} / \mathrm{m})$ and $K_{m} 0.412(\mathrm{Nm} / \mathrm{A})$. The cost function of the optimal control problem is defined by

$$
J(x, u)=\int_{0}^{\infty}\left(x^{T} Q x+u^{T} R u\right) d t,
$$

where $Q=\operatorname{diag}(1,0.5,2,0.05,0.05,1)$ and $T=0.01 \mathrm{~s}$. The algorithm is executed every $2 \mathrm{~s}$. The control objective is minimize both the inclined and directional angles while the robot is tracking the reference. For instance, in the $x$ direction, in the first $10 \mathrm{~s}$, the robot was expected to track at $0 \mathrm{~m}$ from its initial position of $0.5 \mathrm{~m}$. And in the next $10 \mathrm{~s}$, the robot was required to stay at $1 \mathrm{~m}$. To this end, we initially set the state feedback controller as follows,

$$
K_{0}=\left[\begin{array}{cccccc}
-0.2 & -0.8 & -0.3 & -0.2 & -0.1 & -0.2 \\
-0.2 & -0.8 & 0.3 & -0.2 & -0.1 & 0.2
\end{array}\right] \text {. }
$$

And the controller was adaptively updated by the proposed algorithm after every iteration. For instance, at 3 running iterations, the controller was

$$
\begin{aligned}
& K_{3}= \\
& {\left[\begin{array}{cccccc}
-0.189 & -0.617 & -0.489 & -0.178 & -0.078 & -0.231 \\
-0.282 & -0.666 & 0.693 & -0.226 & -0.083 & 0.233
\end{array}\right] .}
\end{aligned}
$$

The simulation results of the two-wheel robot during first 20 $\mathrm{s}$ are demonstrated in Fig. 3, which promise practicality of the proposed approach.

\section{CONCLUSIONS}

An efficient method using reinforcement learning based adaptive dynamic programming to control a two-wheel robot has been presented in the paper. The proposed algorithm guarantees finding the optimal state controller where its convergence has theoretically been proved. The optimal control scheme was successfully implemented in a synthetic robot, where the obtained results promise its practicality.

\section{REFERENCES}

[1] C. Chang and S. Chang, "Using reinforcement learning to achieve two wheeled self balancing control," in 2016 International Computer Symposium (ICS), 2016, pp. 104-107.

[2] H. X. Le, A. V. Le, and L. Nguyen, "Adaptive fuzzy observer based hierarchical sliding mode control for uncertain 2d overhead cranes," Cyber-Physical Systems, vol. 5, no. 3, pp. 191-208, 2019

[3] T. V. Nguyen, N. H. Thai, H. T. Pham, T. A. Phan, L. Nguyen, H. X. Le, and H. D. Nguyen, "Adaptive neural network-based backstepping sliding mode control approach for dual-arm robots," Journal of Control, Automation and Electrical Systems, vol. 30, no. 4, pp. 512-521, Aug 2019.

[4] V.-A. Le, H.-X. Le, L. Nguyen, and M.-X. Phan, "An efficient adaptive hierarchical sliding mode control strategy using neural networks for $3 \mathrm{~d}$ overhead cranes," International Journal of Automation and Computing, vol. 16, no. 5, pp. 614-627, Oct 2019.

[5] D. T. Pham, T. V. Nguyen, H. X. Le, L. Nguyen, N. H. Thai, T. A. Phan, H. T. Pham, A. H. Duong, and L. T. Bui, "Adaptive neural network based dynamic surface control for uncertain dual arm robots," International Journal of Dynamics and Control, vol. 8, no. 3, pp. 824-834, Sep 2020.

[6] H. X. Le, L. Nguyen, and K. Thiyagarajan, "A dynamic surface controller based on adaptive neural network for dual arm robots," in 2020 15th IEEE Conference on Industrial Electronics and Applications (ICIEA), 2020, pp. 555-560.

[7] U. T. T. Hoang, H. X. Le, N. H. Thai, H. V. Pham, and L. Nguyen, "Consistency of control performance in $3 \mathrm{~d}$ overhead cranes under payload mass uncertainty," Electronics, vol. 9, no. 4, p. 657, Apr 2020. [Online]. Available: http://dx.doi.org/10.3390/electronics9040657

[8] Y. Jiang and Z. P. Jiang, Robust Adaptive Dynamic Programming. John Wiley \& Sons, 2017.

[9] S. Bhasin, R. Kamalapurkar, M. Johnson, K. Vamvoudakis, F. Lewis, and W. Dixon, "A novel actor-critic-identifier architecture for approximate optimal control of uncertain nonlinear systems," Automatica, vol. 49, no. 1, pp. 82-92, 2013.

[10] D. Vrabie, O. Pastravanu, M. Abu-Khalaf, and F. Lewis, "Adaptive optimal control for continuous-time linear systems based on policy iteration," Automatica, vol. 45, no. 2, pp. 477-484, 2009.

[11] Y. Jiang and Z.-P. Jiang, "Computational adaptive optimal control for continuous-time linear systems with completely unknown dynamics," Automatica, vol. 48, no. 10, pp. 2699-2704, 2012.

[12] F. L. Lewis, D. Vrabie, and V. L. Syrmos, Optimal control. John Wiley \& Sons, 2012.

[13] L. V. Kantorovich and G. P. Akilov, Funclional analysis in sortned spaces. New York: Mamillan, 1964.

[14] D. Kleinman, "On an iterative technique for riccati equation computations," IEEE Transactions on Automatic Control, vol. 13, no. 1, pp. 114-115, 1968. 